\title{
Noncoding RNAs and Its Implication as Biomarkers in Renal Cell Carcinoma: A Systematic Analysis
}

\author{
Shiv Verma ${ }^{1,2}$, Sanjay Gupta ${ }^{1,2,3,4,5}$
}

Cite this article: Verma S, Gupta S: Noncoding RNAs and its implication as biomarkers in renal cell carcinoma: A systematic analysis. Ann Urol Oncol 2019; 2(2): 47-57. https://doi. org/10.32948/auo.2019.03.28

\begin{abstract}
Renal cell carcinoma (RCC) is one of the most devastating disease with higher mortality rates. It comprises several subtypes exhibiting distinct histological features and clinical staging. Despite recent advancement in understanding the biology of RCC success in treatment rates remains dismal. This may be partly due to lack of specific biomarkers for early detection/prognosis and poor clinical outcome. Noncoding protein transcripts in the genome play important role in the initiation, evolution and progression of cancer. With the advancement in genomic analysis techniques, especially next-generation sequencing, a large number of new transcripts have been discovered, leading to better understanding of coding and noncoding RNAs. In the present review, we summarize recent advancement on renal cancer associated noncoding RNAs which includes long noncoding RNAs, microRNAs, and circular RNAs for their involvement in RCC along with their clinical implication as prognostic and diagnosis biomarkers.
\end{abstract}

Key words renal cell carcinoma, noncoding RNAs, biomarkers, diagnosis, prognosis

1. Department of Urology, Case Western Reserve University, School of Medicine, Cleveland, OH, USA.

2. The Urology Institute, University Hospitals Cleveland Medical Center, Cleveland, OH, USA.

3. Department of Nutrition, Case Western Reserve University, Cleveland, OH, USA.

4. Department of Urology, Louis Stokes Cleveland Veterans Affairs Medical Center, Cleveland, OH, USA.

5. Division of General Medical Sciences, Case Comprehensive Cancer Center, Cleveland, OH, USA.

Correspondence: Sanjay Gupta (Department of Urology, The James and Eilleen Dicke Research Laboratory, Case Western Reserve University, 10900 Euclid Avenue, Cleveland, Ohio 44106, USA; E-mail: sanjay.gupta@case.edu). 


\section{Introduction}

Renal cell carcinoma (RCC) is one of the most devastating cancer in adults, like other malignant diseases, its prognosis and diagnosis in early stage is still difficult [1]. Conventionally, it was considered that nearly all RCC and associated diseases are resistant to chemotherapy and radiation treatment $[2,3]$. At present, the most common treatment of renal cancer is still radiation therapy for the late-stage disease; unfortunately, the response rate is less than $20 \%$ [4]. Metastatic renal cell carcinoma has a very poor prognosis, therefore better understanding of the epigenetics/genomics and its association with the pathogenesis of renal cancer might reveal new opportunities for the discovery of effective cancer biomarkers and therapeutic targets.

Recent development in encoding of the human genome and the Encyclopedia of DNA Elements (ENCODE) project has discovered that most of the human genome is transcribed into RNAs that do not encode proteins [5]. Moreover, the large database genomic information such as The Cancer Genome Atlas (TCGA), and the International Cancer Genome Consortium (ICGC), revealed that dysregulation in genome either due to mutation or copy number changes related to cancer overlap with noncoding DNA elements or noncoding RNA genes [6]. The outcome of above mentioned projects reformed the perception of noncoding (nc) RNAs from 'junk' transcriptional products to functional regulatory molecules [7]. This underlines the fact that junk DNA/RNA is indeed not a wasteland anymore. In fact, genes only comprise $2 \%$ of human genome or encoded proteins and the remaining noncoding portions of the genome transcription products are noncoding RNAs which differ in biogenesis and function. Nearly $80 \%$ of the human genome comprehends regulatory elements as well as ncRNA genes [8]. With the advancement in genomic analysis techniques, especially next-generation RNA sequencing (whole genome/ exosome), a large number of new transcripts have been discovered, leading to better understanding of coding and noncoding RNAs. These include the discovery of long noncoding RNA (lncRNA), microRNAs (miRNAs), circular RNAs (circRNAs) playing a critical role in post-transcriptional gene regulation adding new dimensions to the development of novel diagnostic and treatment tools.

The present review summarize renal cancer-associated noncoding RNAs which includes lncRNAs, miRNAs, and circRNAs their involvement in tumor suppression or tumor promotion along with their clinical implication as prognostic and diagnostic biomarkers. In context to its implication as biomarker we have used 3 independent sets of GEO database viz. expression profiles of miRNAs in clear cell renal cell carcinomas (ccRCCs) and the matched normal kidney tissues (NCTs) by using a miRNAs microarray platform which comprise a total of 851 human miRNAs.

\section{Long noncoding RNA and its mechanistic role in renal cancer}

The widely discovered IncRNA is a novel heterogeneous class of noncoding RNA [9] with more than 200 nucleotides [10]. Since the discovery of lncRNAs, it was obvious that it played an important role in regulating gene expression including chromatin and histone modifications, transcription and post-transcriptional processing [11-13]. It also interacts with RNA binding proteins, and act as co-activator of transcription factors, and inhibit the transcription process [14]. In addition to above, IncRNAs can modulate gene expression at post-transcriptional level or during splicing process of pre-mRNA, which could be linked with cancer $[15,16]$.

LncRNAs are defined by length ( > 200nt) which commonly originate from intergenic regions and are transcribed by RNA polymerase II. Applied next-generation sequencing have provided accumulating evidence of IncRNA deregulation in human cancers. In context to its regulatory mechanism, lncRNA is involved in 3 major physiological roles including transcriptional regulation, post-transcriptional regulation and chromatin remodeling (Figure 1A). Moreover, the mechanistic role of lncRNAs was decipher earlier in breast cancer where notable increase in lncRNAsHOTAIR promotes invasion of breast carcinoma cells [17]. The above investigation represents a role model of lncRNAs and its regulatory function in renal cancer. Biological roles of lncRNAs include chromatin modeling where 5'domain of 1ncRNAsHOTAIR interacts with Polycomb Repressive Complex 2 (PRC2) complex subunits viz. Enhancer of Zeste Homologue 2 (EZH2) and Suppressor of Zeste 12 (SUZ12) leading to alteration in the histone $\mathrm{H} 3$ lysine-27 trimethylation resulting in epigenetic modification of genes thereby increasing cancer invasiveness and progression [17, 18] (Figure 1B \& C). Other mechanism(s) also exist, in particular, lncRNAs and its regulatory role in renal cancer. For instance, the role of IncRNA-SARCC and its function as a tumor suppress or in regulating androgen receptor in renal cell carcinoma is demonstrated by repressing AR activity through physical interaction (Figure 1C). LncRNA-SARCC binds and destabilize AR protein inhibiting AR function leading
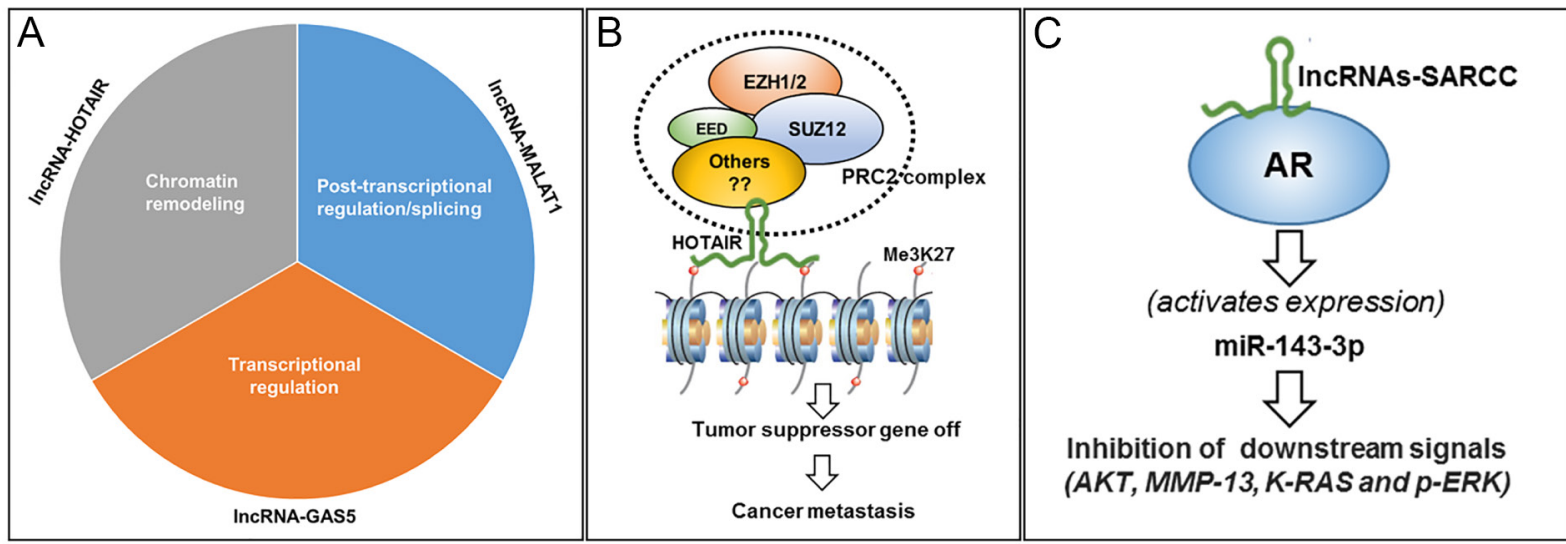

Figure 1. Schematic representation of IncRNAs and their mechanistic regulatory role in cancer. A: Regulatory role of some select IncRNA; B: Presentation of IncRNAs HOTAIR binding with PRC2 complex and modulating the regulation of histone. This event leads to turn-off the tumor suppressor gene thereby increasing cancer metastasis; C: During hypoxia, IncRNAs-SARCC binds to androgen receptor (AR) and deactivates AR and modulate post-transcription. Consequently, HIF2A (hypoxia responsive element) binds to the promoter region on IncRNAs-SARCC and results in the upregulation of cMYC increasing cell proliferation. 
Table 1. List of IncRNA proposed as prognostic biomarker and therapeutic target in renal cancer.

\begin{tabular}{|c|c|c|c|}
\hline LncRNA name & Population cohort & Clinical utility & Reference \\
\hline CADM1-AS1 & China & Prognostic biomarker & {$[28]$} \\
\hline CCAT1 & China & Therapeutic target & [57] \\
\hline COL18A1-AS1 & China & Prognostic biomarker & {$[58]$} \\
\hline DNM1P35 & China & Prognostic biomarker & [59] \\
\hline DRAIC & USA & Prognostic biomarker & {$[33]$} \\
\hline DUXAP8 & China & Prognostic biomarker & [35] \\
\hline EGOT & China & Prognostic biomarker & {$[60]$} \\
\hline EPB41L4A-AS2 & China & Prognostic biomarker & [34] \\
\hline FENDRR & China & Therapeutic target & [61] \\
\hline FILNC1 & China & Prognostic biomarker & [62] \\
\hline H19 & China & Prognostic biomarker & [63] \\
\hline LINC00152 & China & Prognostic biomarker & {$[64]$} \\
\hline lnc-ZNF180-2 & Germany & Prognostic biomarker & {$[31]$} \\
\hline LUCAT1 & China & Biomarker/Therapeutic target & [65] \\
\hline MALAT1 & China & Diagnosis and prognosis & [21] \\
\hline MIR155HG & China & Prognostic biomarker & [59] \\
\hline NBAT-1 & China & Biomarker/Therapeutic target & [29] \\
\hline NEAT1 & China & Prognostic biomarker & {$[66]$} \\
\hline NONHSAT123350 & China & Therapeutic target & {$[32]$} \\
\hline OTUD6B-AS1 & China & Therapeutic target & [67] \\
\hline PIK3CD-AS1 & China & Prognostic biomarker & [49] \\
\hline PVT1 & China & Diagnosis and prognosis & {$[68]$} \\
\hline RCCRT1 & China & Biomarker/Therapeutic target & {$[23]$} \\
\hline ROR & China & Therapeutic target & [69] \\
\hline SARCC & China & Therapeutic target & {$[70]$} \\
\hline SLC25A5-AS1 & China & Prognostic biomarker & [19] \\
\hline SLINKY & Japan & Prognostic biomarker & {$[71]$} \\
\hline TREML3P & China & Prognostic biomarker & [49] \\
\hline TUG1 & China & Prognostic biomarker & {$[72]$} \\
\hline UCA1 & China & Prognostic biomarker & {$[73]$} \\
\hline XIST & China & Therapeutic target & {$[74]$} \\
\hline PANDAR & China & Prognostic biomarker & {$[75]$} \\
\hline SRLR & China & Biomarker/Therapeutic target & {$[76]$} \\
\hline
\end{tabular}


Table 1. List of IncRNA proposed as prognostic biomarker and therapeutic target in renal cancer (Continued).

\begin{tabular}{llll}
\hline LncRNA name & Population cohort & Clinical utility & Reference \\
\hline Z38 & China & Diagnostic biomarker & {$[77]$} \\
CASC2 & China & Diagnostic biomarker & {$[78]$} \\
SPRY4-IT1 & China & Prognostic/Therapeutic target & {$[24]$} \\
SDPR-AS & China & Biomarker/Therapeutic target & {$[79]$} \\
HOTTIP & China & Therapeutic target & {$[80]$} \\
\hline
\end{tabular}

to transcriptionally activate miR-143-3p expression, consequently inhibiting the downstream signal including AKT, MMP-13, K-RAS and P-ERK (Figure 1C).

\section{Long noncoding RNAs as biomarker in renal cell carcinoma}

Identification for potential biomarkers in RCC, we downloaded expression profiles of lncRNAs, miRNAs, and mRNAs from The Cancer Genome Atlas (TCGA) database. The results of survival and regression analysis indicated 6 differentially expressed lncRNAs (DElncRNAs) named as COL18A1-AS1, WT1-AS, LINC00443, TCL6, AL356356.1 and SLC25A5-AS1 which significantly correlate with the clinical traits of RCC patients and might serve as biomarker(s) in RCC [19]. Another class of gene named as "alpha gene" or MALAT1 located on chromosome 11q13 reported to be up-regulated in several human cancers including lung, breast, pancreas, liver, colon, uterus, cervix and prostate [20]. Expression of MALAT1 in human RCC tissue was associated with reduced patient survival. Silencing of MALAT1 decreased RCC cell proliferation, invasion and also induced apoptosis in these cancer cells [21]. Mechanistic investigations showed that MALAT1 was transcriptionally activated by c-Fos and that it interacts with EZH2 in RCC [21]. Overexpression of MALAT1 confers oncogenic potential in RCC and may serve as a novel biomarker for renal cancer [21, 22]. Several other IncRNAs were identified who's dysfunction lead to the renal cancer cell proliferation, invasion and migration. In particular, increased expression of RCCRT1 [23], SPRY4-IT1 [24], H19 [25], and MALAT1 [21, 26], HOTTIP [27] were associated with poor prognosis. Whereas decrease expression of CADM1-AS1 [28], NBAT-1 [29, 30], lncZNF180-2 [31], NONHSAT123350 [32], downregulate RNA in androgen independent cells (DRAIC) [33], and EPB41L4AAS2 [34] were related to poor prognosis (Table 1). The newly discovered pseudogene-derived lncRNA named DUXAP8, a 2107bp RNA, was markedly upregulated in RCC [35]. Deciphering the molecular and biological mechanism(s) suggested that lncRNA DUXAP8 enhanced RCC progression through downregulating miR-126, which opens new prognostic approach for the treatment of RCC[35]. These findings emphasize clinical implication of lncRNAs in early diagnostic/prognostic biomarker in renal cancer.

\section{MiRNA in renal cancer}

Another class of noncoding RNAs is categorized as small RNA or microRNAs (miRNAs). This particular class of noncoding RNA is 20-23 base pair nucleotide long, and functionally suppresses the expression of gene by binding to the 3' or 5' UTR region [36]. MiRNAs may target many different genes based on the presence of complementary mRNA targets for miRNA seed sequences. Several experimental studies reveal the fact that dysregulation of miRNAs may be linked to cancer cell proliferation and/or tumor progression. Conventionally, it was perceived that increased/ decreased expression of miRNAs function as tumor suppressors modulating the expression of genes/transcription factors. The mechanisms underlying the dysregulation of miRNAs varies in cancer which includes changes in the transcriptional regulation of miRNAs by tumor-related protein viz. HIF $\alpha$, c-myc, EZH2, Notch1, and mutation in DICER, a protein involved in miRNA processing [37].

The noncoding RNA profile array of clear cell (cc) RCC was downloaded from GEO database (GSE116251 platform IDGPL25243) and data was reanalyzed using R and GEO2R script. The expression of 800 miRNAs were assessed in paired tumor and normal tissues from a discovery cohort of $18 \mathrm{ccRCC}$ patients via Nanostring assay platform. Analysis of miRNA expression profiles of 36 samples revealed a subset of 3 miRNAs with $\log 2$ fold change ( $\log 2 \mathrm{FC}$ ), which include has-miR-1246, has-miR-592, has-miR-21-5p, and has-miR-155-5p (Additional file 1: Table S1). In terms of downregulated miRNA, the expression of miR-200c$3 p$ was -2.3 fold down in the above mentioned dataset. Moreover, dichotomized analysis linked the expression of 2 miRNAs, miR155-5p and miR-210-3p with ccRCC recurrence [38].

Another set of miRNA expression profile (GSE95385, platform ID-GPL16770) in clear cell papillary renal cell carcinoma compared to normal adjacent tissue, identified 14 miRNAs (Additional file 2: Table S2), which showed upregulated

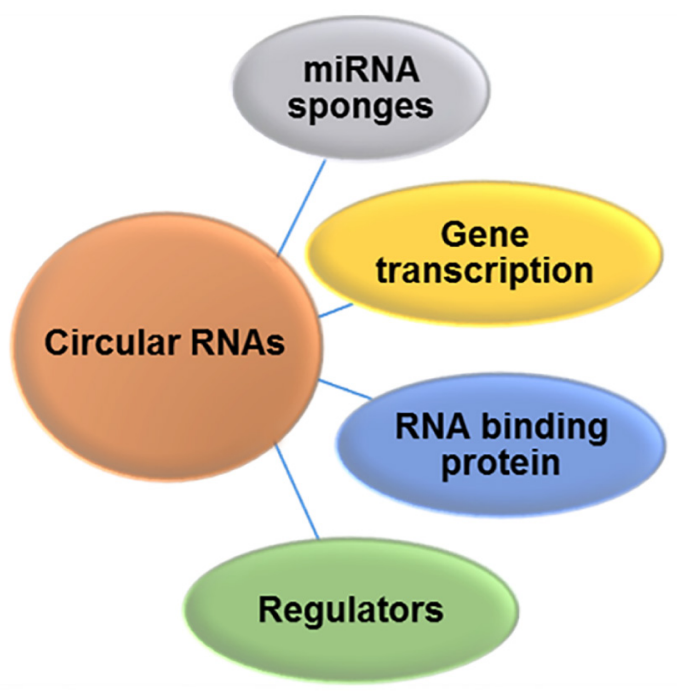

Figure 2. Functional role of circular RNA in gene regulation. Circular RNA serve as regulator in gene expression by competing with mRNA production in pre-miRNA splicing, and as miRNA sponges, interacting with RNA binding proteins and gene regulators. 
expression greater than $\log 2$ fold 1.5. Among them the expression of hsa-miR-204, miR-192, miR194, was 5.3, 3.65 and $3.61 \log 2$ fold higher and the expression of miR-210, miR-21, miR-34a and miR122 exhibited downregulation at $-4.2,-2.4,-2.75$, and -2.64 $\log 2$ fold, respectively (Table 2 ).

In another array dataset (GSE71302, platform ID-GPL10850) of ncRNA profiling, the expression profile of miRNAs in human ccRCCs, compared with normal kidney tissues (NCTs) identified a total of 851 miRNAs. In results, 22 miRNAs high $\log 2 \mathrm{FC} \mathrm{(}>$ 2.0), among them the expression of hsa-miR-141, and hsa-miR200c was significantly downregulated in ccRCC specimens [39]. On the other hand, 13 miRNAs showed increased level of expression in ccRCC specimens, among them the expression of hsa-miR-122, hsa-miR-210, and hsa-miR-224 (Table 2), the other 11 hsa-miRNAs are listed in the Additional file 3: Table S3. The above mentioned dataset were pooled and reanalyzed (using $\log 2 \mathrm{FC}$ filter) largest to smallest, $31 \mathrm{miRNAs}$ were upregulated and differentially expressed ( $>\log 2 \mathrm{FC}, 2$ to 6 ) in RCC patient's samples. Among them, the expression of hsa-miR-141 showed highest level of expression $(6.70, \log 2 \mathrm{FC})$, followed by hsa-miR$200 \mathrm{c}(4.9, \log 2 \mathrm{FC})$, has-miR-138 $(4.51, \log 2 \mathrm{FC})$ and hsa-miR-210 (4.26, $\log 2 \mathrm{FC})$, respectively.

\section{MiRNAs as biomarker in renal cancer}

Previously, we have documented the role of miRNA as prognostic/ diagnostic biomarker in prostate cancer [40]. Here we review the role of miRNA as prognostic biomarker in RCC which is examined by metadata analysis. The above mentioned metadata analysis from 3 independent cohorts in renal cancer, demonstrate that miR-210 was upregulated ( $\log$ FC 4.25/GSE95385; $\log 2 \mathrm{FC} 4.4 /$ GSE71302) in ccRCC despite different array platforms (GPL16770/ GPL10850). The high expression of miR-210 in renal cancer further supported in RCC patients samples when compared with normal tissue controls, miR-210 was overexpressed over 10-fold higher in the ccRCCs $(\mathrm{N}=32, \mathrm{P}<0.001$, Mann-Whitney test), 2.8fold in the papillary tumors ( $\mathrm{N}=9, \mathrm{P}<0.05$, Mann-Whitney test). This set of information including metadata analysis in independent cohorts suggest miR-210 as prognostic biomarker in ccRCC. With respect to miRNA: mRNA target interaction, it was revealed that miR-210 binds to the $3^{\prime}$ UTR region of hypoxia-inducible factors (HIFs) and modulate gene expression of HIF1and HIF2 at the post-transcriptional level [41]. The experimentally validated in vivo study showed high expression of miR-210 association with improved clinico-pathological prognostic factors.

\section{Circular RNAs in renal cancer}

A new class of noncoding RNAs referred as circular RNA provides new optimism in the field of cancer research. CircRNAs generated during the process of splicing of pre-mRNA from the coding/ noncoding genomic region or both, with a majority comprising of exonic RNAs. CircRNA skip the process of canonical splicing and is formed by non-sequential back-splicing of pre-messenger RNA (pre-mRNA) transcripts [42]. Functionally, circRNAs acts as miRNA sponge combining with RNA binding proteins (RBPs) and is operational as a transcription factor and translation of proteins. Another novel role of circRNA is to compete with endogenous RNA. Together circRNA with miRNAs can influence the stability of target gene inhibitors and in regulating gene expression at

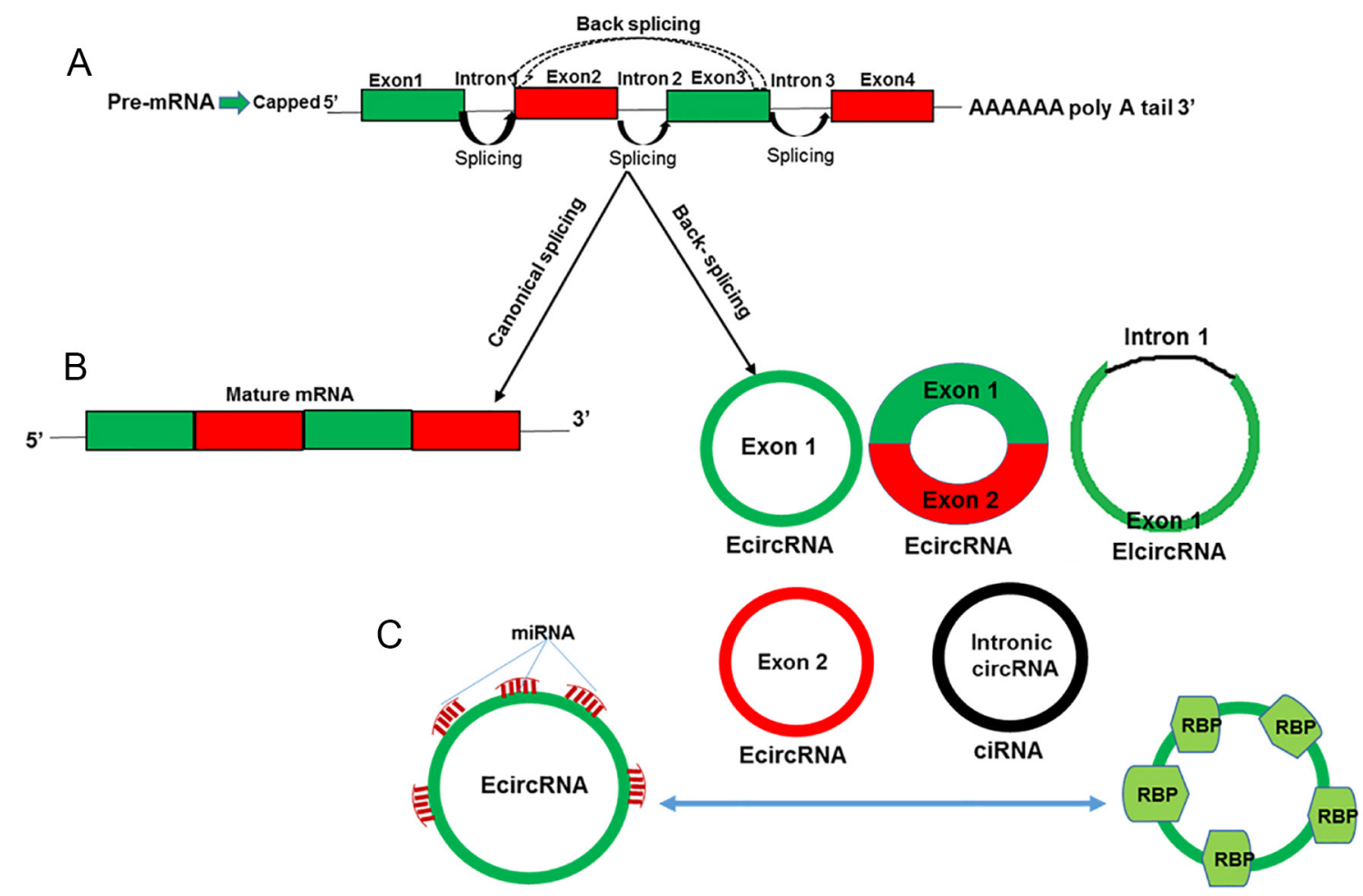

Figure 3. Mechanism by which circular RNAs play important roles as miRNA sponges, gene transcription and gene regulators, RNA-binding protein (RBP) sponges and protein/peptide translators. A: Schematic representation of circular RNA biogenesis: Canonical pre-mRNA splicing yielded mature mRNA; B: Pre-mRNA undergoes the process called back-splicing and circularization, which resulted in the formation of intronic circRNAs, exonic circRNAs and exonic-intron circRNAs; C: Functional role of circRNA acting as miRNA sponges by competing for miRNA binding sites, diminishing the effect of miRNAmediated regulatory activities by binding to RBPs. 
Table 2. List of miRNAs and its expression associated with renal cancer.

\begin{tabular}{|c|c|c|c|}
\hline MicroRNA & Expression & Target gene & Gene full name \\
\hline $\operatorname{miR}-100$ & Down & RBX1 & Ring-box 1, E3 ubiquitin protein ligase \\
\hline miR-10b & Down & $\mathrm{BDNF}$ & Brain-derived neurotrophic factor \\
\hline $\operatorname{miR}-125 b$ & Down & RP1-228P16.5 & Uncharacterized protein \\
\hline $\operatorname{miR}-26 \mathrm{a}+$ & Down & POLR3G & RNA polymerase III (DNA directed) polypeptide G (32kD) \\
\hline miR-133b & Down & LHFP & Lipoma HMGIC fusion partner \\
\hline miR-135b & Down & ZNF302 & Zinc finger protein 302 \\
\hline miR-136 & Down & MEX3C & Mex-3 RNA binding family member C \\
\hline miR-141 & Down & ZNF385D & Zinc finger protein $385 \mathrm{D}$ \\
\hline miR-149 & Down & LRIG2 & Leucine-rich repeats and immunoglobulin-like domains 2 \\
\hline miR-154 & Down & $\mathrm{E} 2 \mathrm{~F} 5$ & E2F transcription factor 5, p130-binding \\
\hline miR-199a & Down & KLHL3 & Kelch-like family member 3 \\
\hline miR-200a* & Down & ZEB1 & Zinc finger E-box binding homeobox 1 \\
\hline $\operatorname{miR}-200 \mathrm{~b}$ & Down & ZEB1 & Zinc finger E-box binding homeobox 1 \\
\hline $\operatorname{miR}-200 \mathrm{c}$ & Down & ZEB1 & Zinc finger E-box binding homeobox 1 \\
\hline $\operatorname{miR}-211$ & Down & AP1S2 & Adaptor-related protein complex 1 , sigma 2 subunit \\
\hline miR-30a-3p & Down & DST & Dystonin \\
\hline $\operatorname{miR}-337$ & Down & RAP2B & RAP2B, member of RAS oncogene family \\
\hline $\operatorname{miR}-377$ & Down & SLC6A19 & Solute carrier family 6 (neutral amino acid transporter), member 19 \\
\hline $\operatorname{miR}-411$ & Down & ATP6V1G1 & ATPase, $\mathrm{H}+$ transporting, lysosomal $13 \mathrm{kDa}, \mathrm{V} 1$ subunit $\mathrm{G} 1$ \\
\hline $\operatorname{miR}-429$ & Down & ZEB1 & Zinc finger E-box binding homeobox 1 \\
\hline $\operatorname{miR}-507$ & Down & ZNF681 & Zinc finger protein 681 \\
\hline $\operatorname{miR}-510$ & Down & $\mathrm{CMC} 2$ & COX assembly mitochondrial protein 2 homolog (S. cerevisiae) \\
\hline $\operatorname{miR}-514$ & Down & GMFB & Glia maturation factor, beta \\
\hline miR-142-3p & Up & HSBP1 & Heat shock factor binding protein 1 \\
\hline miR-155 & Up & $\mathrm{H} 3 \mathrm{~F} 3 \mathrm{~A}$ & $\mathrm{H} 3$ histone, family $3 \mathrm{~A}$ \\
\hline $\operatorname{miR}-185$ & Up & PRRT2 & Proline-rich transmembrane protein 2 \\
\hline $\operatorname{miR}-21$ & Up & FGF18 & Fibroblast growth factor 18 \\
\hline $\operatorname{miR}-224$ & Up & INIP & INTS3 and NABP interacting protein \\
\hline $\operatorname{miR}-34 a$ & Up & MDM4 & Mdm4 p53 binding protein homolog (mouse) \\
\hline $\operatorname{miR}-34 b$ & Up & FDX1 & Ferredoxin 1 \\
\hline MiR-708 & $\mathrm{Up}$ & ATP6V1G1 & ATPase, $\mathrm{H}+$ transporting, lysosomal $13 \mathrm{kDa}, \mathrm{V} 1$ subunit $\mathrm{G} 1$ \\
\hline miR-1285 & Up & MRPL44 & Mitochondrial ribosomal protein L44 \\
\hline $\operatorname{miR}-221$ & Up & CDKN1B & Cyclin-dependent kinase inhibitor 1B (p27, Kip1) \\
\hline
\end{tabular}


Table 2. List of miRNAs and its expression associated with renal cancer (Continued).

\begin{tabular}{llll} 
MicroRNA & Expression & Target gene & Gene full name \\
\hline miR-21 & Up & FGF18 & Fibroblast growth factor 18 \\
miR-183 & Up & UBE2G1 & Ubiquitin-conjugating enzyme E2G 1 \\
miR-135a & Down & c-MYC & MYC binding protein \\
miR-218 & Down & TUB & Tubby bipartite transcription factor \\
miR-205 & Up & Src family & Src family \\
miR-203 & Down & FGF2 & Fibroblast growth factor 2 \\
miR-210 & Up & FGF1R & Fibroblast growth factor receptor-like 1 \\
\hline
\end{tabular}

transcriptional level [43, 44].

Circular RNAs are endogenous non-coding RNA, and the role of circRNA was pivotal in terms of regulating diverse cellular process, which includes gene transcription, RNA binding protein and acts as regulators and miRNAs sponge (Figure 2). In terms of biogenesis, circRNAs are generated by a spliceosome mediated pre-mRNA back splicing, fundamentally different than regular canonical (linear) splicing (Figure 3A). Regarding their biogenesis from different genomic regions (exon/intron) circRNA can be categorized into four types including i) exonic circular RNA (EcircRNAs), ii) circular intronic RNA (ciRNAs), iii) exon-intron circular RNAs (EIciRNAs), and iv) intergenic circular RNAs (Figure 3B). CircRNAs has better ability to bind with miRNAs and thus called as "super sponge" [45-47]. The mechanism of regulation of circRNA is similar like miRNAs as it blocks the binding of miRNAs and directly binds to the miRNAs indirectly regulating gene expression [43]. The mechanistic undermined function of cirRNA is that it plays as regulator of gene expression by competing with mRNA production in pre-miRNA splicing. Moreover, circular RNAs can serves as mRNA traps, another form of alternate splicing, and remove the start codons from mature mRNAs to reduce protein translation in cancer.

Remarkably, interaction between circRNA and miRNAs has already been observed to perform a significant role in a variety of human cancers. However, the current knowledge about the involvement of circRNAs in cancer development and progression is limited, and the role of circRNAs as miRNA sponge has been proposed as the most frequent mechanism of circRNA activity in tumor cells (Figure 3C). CircRNA act like miRNA sponge by competing for miRNA binding sites (Figure 3C), and reduce the effect of miRNA-mediated regulatory activities such as posttranscriptional repression. In fact, overexpression of miRNA sponge acting circRNAs increases the expression, whereas silencing of these circRNAs decreases the expression of miRNA targets. The "super sponge" mechanism ruled among the other proposed mechanism, indeed circRNAs serves as miRNA sponge with suggested potential role as competitive endogenous RNAs competing for miRNA-binding sites, thus affecting miRNA activities. CircRNA (circHIPK3) was observed to sponge 9 miRNAs with 18 potential binding sites and in particular regulates cell growth by sponging multiple miR-124 and inhibiting miR-124 activity in malignant tumors [48].

Several circRNAs have been identified so far with reference to renal cancer. Recently, it was shown that expression of circRNA, $\mathrm{H}$ long terminal repeat-associating protein 2 (HHLA2) was increased in renal cancer tissues compared with normal renal tissue both at the transcriptional and protein level [49]. Another, novel circRNA,
circPCNXL2 was significantly upregulated in renal cancer. Indeed high abundance of circPCNXL2 was directly linked with poor survival rate of RCC patients [50]. Knockdown of circPCNXL2 has shown to reduceRCC cell proliferation in invitro and in vivo studies. Mechanistically, circPCNXL2 acts as miRNA sponge of miR-153 and modulate the expression of ZEB2 target gene, and thus increase cancer cell proliferation and invasion. Xiong et al. identified a new circRNA (circRNA ZNF609) whose expression is significantly increased in RCC. CircRNA ZNF609 acts as miRNA sponge of miRNA-138-5p and modulate the expression of forkhead box P4 (FOXP4), ultimately influencing renal cancer cell proliferation. Another circular RNA (circATP2B1) also influences renal cancer cell proliferation via miR-204-3p, and increases the expression of fibronectin 1 (FN1) [51]. Wang et al. identified a new circular RNA (circHIAT1) that was downregulated in ccRCC tissues [25]. Functionally, CircHIAT1 acts as miRNA sponge of miR-195-5p/29a-3p/29c-3p and regulates the expression of CDC42. Activation of androgen receptor suppressed circHIAT1 expression resulting in decreased CDC42 expression and enhanced ccRCC cell migration and invasion [25].

\section{Circular RNAs as biomarker in renal cancer}

Emerging evidences deciphered the role of circRNA in cancer having potential to serve as biomarker and therapeutic target in cancer. In context to biomarker, circRNAs stably expressed in saliva, blood, and exosomes, which satisfy the criteria as cancer biomarkers [52]. Also because circRNA has no open linear tail like other ncRNA and therefore are insensitive to exonuclease. Thus circRNAs are enriched and stable in exosomes also referred as exo-circRNAs [53], and exosome secretion may represent one of the mechanisms for the removal of circular RNAs. Quantifying and analyzing circRNAs by RT-PCR and in-situ hybridization is more sensitive compared to protein by an antigen-antibody reaction. For example, it was shown that expression of circular RNA-HHLA2 was increased in ccRCC tissues, compared with normal renal tissues at both transcriptional and protein level and suggested the role of HHLA2 as prognostic biomarker in RCC [54]. Also circular RNA (Hsa_circ_0001451) might be involved in renal tumor progression and suggested its role as prognostic biomarker, however the experimental validation has not been conducted, hence needs further investigation. Another class of circRNA (circATP2B1) also influenced the progression of ccRCC. Mechanistically, circATP2B1 acts as miRNA sponge of miR-204$3 p$ and influence the expression of estrogen receptor beta (ER $\beta)$, together they influence RCC progression, suggesting circRNA ATP2B1 as prognostic biomarker for renal disease [51]. The 
Table 3. Clinical trials on miRNA in renal cancer.

\begin{tabular}{|c|c|c|c|}
\hline noncoding RNA & Clinical trial ID & Description & Status \\
\hline miRNA & NCT00743054 & $\begin{array}{l}\text { The study examines RCC related miRNA and the target genes of } \\
\text { related miRNA and the relationship between RCC related miRNA, } \\
\text { pathological types, tumor stage and prognosis. The purpose of this } \\
\text { study is to investigate the role of miRNA as novel biomarker(s) in } \\
\text { the formation of RCC. }\end{array}$ & Completed \\
\hline miRNA & NCT00806650 & $\begin{array}{l}\text { The study intends to develop a blood test for anti-IMP } 3 \text { antibody } \\
\text { and microRNA in serum and tissue samples to diagnose RCC and } \\
\text { provide effective treatment options to patients. }\end{array}$ & Completed \\
\hline miRNA & NCT01829971 & $\begin{array}{l}\text { Phase I, open-label, multicenter, dose-escalation study } \\
\text { investigates the safety, pharmacokinetics and pharmacodynamics } \\
\text { of the microRNA, MRX } 34 \text { in patients with unresectable primary } \\
\text { liver cancer or advanced or metastatic kidney cancer. }\end{array}$ & Terminated \\
\hline
\end{tabular}

expression of circPCNXL2 was significantly increased in RCC patients, indeed the higher expression corresponds to poor survival of ccRCC patients [50]. Quantitative expression analysis of A-498, AXHN, ACHN, OS-RC-2, 769-P and G-401 as numerous renal cell lines showed increased expression of circ-ZNF609 [55].

\section{Clinical implication of noncoding RNA and renal cancer}

It is clear from the above information that several research groups have identified ncRNA and proposed as prognostic/diagnostic biomarker in renal cancer. However, the lab to clinic data is not very striking. Using the web portal (https://clinicaltrials.gov) with key words "Renal Cancer" and "Noncoding RNA/miRNAs/ lncRNAs" and "Circular RNA" to search the clinical impact of ncRNA exhibited a small list of clinical trials particularly with miRNA in renal cancer (Table 3). Moreover, the direct clinical implication of ncRNA against renal cancer is not documented so far. As mentioned above, targeting exosome cargos may express high diagnostic and/or prognostic potential. The current information presented here emphasize the role of ncRNAs as prognostic marker in renal cancer. Indeed, the role of circRNA was inevitable in terms of its existence in exosomes. In 2015, Lerner et al. first identified circRNA in exosomes from the RNA-Seq data (genome wide) enriched in exosomes compared to parental cells [56]. Clinical trials implementing exosome research is needed in future on RCC.

\section{Conclusions and future direction}

Emerging evidence suggests that ncRNAs are a group of sensitive and specific novel noninvasive biomarkers for the prediction of pathological grade, metastasis/recurrence, and survival having significant impact on our understanding of the pathogenesis of RCC. At the same time, ncRNAs have shown to have potential as a tool for early diagnosis and prognosis for RCC. Noncoding RNAs contribute to RCC development at various stages and it is evident that these molecules can target signaling pathways related to RCC pathogenesis. However, dysregulation of the ncRNAs can be either a cause or an effect of carcinogenesis which needs further investigation. Additionally, mechanistic studies this far has provided the rationale of using ncRNAs as potential therapeutic targets in cancer. The bioinformatics and systems biology methods will be useful to investigate the functional roles of ncRNAs in renal cancer cell types to reveal its regulatory mechanism(s). This cell-type specific integrative network analysis will be valuable in understanding ncRNA function(s) in biological and cellular processes, and their specific regulatory roles in RCC.

Advancement in technology and investigations on ncRNAs have drawn increasing attention. The functions of lncRNA in pathogenesis, miRNA in regulation of gene expression and circRNAs as miRNA sponges and transcriptional regulators are increasingly recognized for their roles in biogenesis and functional mechanisms. These findings elucidate the physiological and pathological processes of a number of ncRNAs which may be considered as novel diagnostic biomarkers and potential therapeutic targets in cancer, however further research is needed to elucidate specific molecular mechanisms and pathways connections in relation to RCC. Additional studies are required to accurately identify the mechanisms by which miRNAs affect RCC. Summarizing recent knowledge, ncRNAs represent a new group of molecules having potential for development as tumor biomarkers that may improve the diagnostic, prognostic and even predictive abilities, and finally RCC patient management.

\section{Acknowledgments}

The authors acknowledge the efforts for this article supported by the Department of Veteran Affairs award 1I01BX002494 and Department of Defense grant W81XWH-18-1-0618 and W81XWH-15-1-0558 to SG.

\section{Funding}

This research did not receive any specific financial support from funding agencies in the public, commercial, or not-for-profit sectors.

\section{Ethical policy}

No research involving experimentation on human or animal subjects was conducted.

\section{Author contributions}

SV prepared the draft, collected data from the open source/public domain and performed analysis. SG conceived the manuscript outline, provided input and critical revisions on the manuscript. 


\section{Competing interests}

The authors declare no conflict of interest with the work.

\section{Ethical statement}

Both authors have been personally and actively involved in substantive work leading to the manuscript, and will hold themselves jointly and individually responsible for its content.

\section{Additional files}

Additional file 1: Table S1. GSE116251 list of microRNA expression profile in clear cell renal cell carcinoma (RCC) tissues, analyzed by GEO2R. The adjusted $\mathrm{p}$ value was calculated along with t-test (Moderated t-statistic (only available when two groups of Samples are defined) and B-statistic or log-odds that the gene is differentially expressed (only available when two groups of Samples are defined). The expression values were represented in the form of $\log 2$-fold change between two experimental conditions.

Additional file 2: Table S2. GSE95385 list of microRNA expression profile in clear cell papillary renal cell carcinoma compared to normal adjacent tissue analyzed by GEO2R. The adjusted $p$ value was calculated, along with t-test (Moderated t-statistic (only available when two groups of Samples are defined) and B-statistic or log-odds that the gene is differentially expressed (only available when two groups of Samples are defined). The expression values were represented in the form of $\log 2$-fold change between two experimental conditions.

Additional file 3: Table S3. GSE71302 list of miRNAs in clear cell renal cell carcinomas (ccRCCs) and in matched normal kidney tissues (NCTs), analyzed by GEO2R. The adjusted $p$ value was calculated, along with t-test (Moderated t-statistic (only available when two groups of Samples are defined) and B-statistic or logodds that the gene is differentially expressed (only available when two groups of Samples are defined). The expression values were represented in the form of Log2-fold change between two experimental conditions.

\section{References}

1. Hsieh JJ, Purdue MP, Signoretti S, Swanton C, Albiges L, Schmidinger M, Heng DY, Larkin J, Ficarra V: Renal cell carcinoma. Nature Reviews Disease Primers 2017, 3: 17009.

2. Nathan PD, Eisen TG: The biological treatment of renal-cell carcinoma and melanoma. Lancet Oncol 2002, 3(2): 89-96.

3. Penticuff JC, Kyprianou N: Therapeutic challenges in renal cell carcinoma. American journal of clinical and experimental urology 2015, 3(2): 77-90.

4. Anselmo Da Costa I, Rausch S, Kruck S, Todenhöfer T, Stenzl A, Bedke J: Immunotherapeutic strategies for the treatment of renal cell carcinoma: Where will we go? Expert Review of Anticancer Therapy 2017, 17(4): 357-368.

5. Ecker JR, Bickmore WA, Barroso I, Pritchard JK, Gilad Y, Segal E: Genomics: ENCODE explained. Nature 2012, 489(7414): 52-55.

6. Shukla GC, Gupta S: Hallmarks of cancer- focus on RNA metabolism and regulatory noncoding RNAs. Cancer Letters 2018, 420: 208-209.

7. Doolittle WF: Is junk DNA bunk? A critique of ENCODE. Proceedings of the National Academy of Sciences 2013, 110(14): 5294-5300.

8. Consortium TEP: An integrated encyclopedia of DNA elements in the human genome. Nature 2012, 489: 57-74.
9. Fatica A, Bozzoni I: Long non-coding RNAs: new players in cell differentiation and development. Nat Rev Genet 2014, 15(1): 7-21.

10. Misawa A, Takayama KI, Inoue S: Long non-coding RNAs and prostate cancer. Cancer Sci 2017, 108(11): 2107-2114.

11. Quinodoz S, Guttman M: Long noncoding RNAs: an emerging link between gene regulation and nuclear organization. Trends Cell Biol 2014, 24(11): 651-663.

12. Sun W, Yang Y, Xu C, Guo J: Regulatory mechanisms of long noncoding RNAs on gene expression in cancers. Cancer Genet 2017, 216-217: 105-110.

13. Dykes IM, Emanueli C: Transcriptional and Post-transcriptional Gene Regulation by Long Non-coding RNA. Genomics Proteomics Bioinformatics 2017, 15(3): 177-186.

14. Mathy NW, Chen XM: Long non-coding RNAs (lncRNAs) and their transcriptional control of inflammatory responses. J Biol Chem 2017, 292(30): 12375-12382.

15. Engreitz JM, Haines JE, Perez EM, Munson G, Chen J, Kane M, McDonel PE, Guttman M, Lander ES: Local regulation of gene expression by lncRNA promoters, transcription and splicing. Nature 2016, 539(7629): 452-455.

16. Shukla GC, Singh J: Mutations of RNA splicing factors in hematological malignancies. Cancer Letters 2017, 409: 1-8.

17. Gupta RA, Shah N, Wang KC, Kim J, Horlings HM, Wong DJ, Tsai MC, Hung T, Argani P, Rinn JL et al: Long non-coding RNA HOTAIR reprograms chromatin state to promote cancer metastasis. Nature 2010, 464(7291): 1071-1076.

18. Liu Y-L, Gao X, Jiang Y, Zhang G, Sun Z-C, Cui B-B, Yang Y-M: Expression and clinicopathological significance of EED, SUZ12 and EZH2 mRNA in colorectal cancer. Journal of Cancer Research and Clinical Oncology 2015, 141(4): 661-669.

19. Yang K, Lu XF, Luo PC: Identification of Six Potentially Long Noncoding RNAs as Biomarkers Involved Competitive Endogenous RNA in Clear Cell Renal Cell Carcinoma. 2018, 2018: 9303486.

20. Zhao M, Wang S, Li Q, Ji Q, Guo P, Liu X: MALAT1: A long noncoding RNA highly associated with human cancers. Oncology letters 2018, 16(1): 19-26.

21. Hirata H, Hinoda Y, Shahryari V, Deng G, Nakajima K, Tabatabai ZL, Ishii N, Dahiya R: Long Noncoding RNA MALAT1 Promotes Aggressive Renal Cell Carcinoma through Ezh2 and Interacts with miR-205. Cancer Res 2015, 75(7): 1322-1331.

22. Tian X, Xu G: Clinical value of lncRNA MALAT1 as a prognostic marker in human cancer: systematic review and meta-analysis. BMJ Open 2015, 5(9): e008653.

23. Song S, Wu Z, Wang C, Liu B, Ye X, Chen J, Yang Q, Ye H, Xu B, Wang L: RCCRT1 is correlated with prognosis and promotes cell migration and invasion in renal cell carcinoma. Urology 2014, 84(3): 730.e731-737.

24. Zhang HM, Yang FQ, Yan Y, Che JP, Zheng JH: High expression of long non-coding RNA SPRY4-IT1 predicts poor prognosis of clear cell renal cell carcinoma. Int J Clin Exp Pathol 2014, 7(9): 5801-5809.

25. Wang D, Ding L, Wang L, Zhao Y, Sun Z, Karnes RJ, Zhang J, Huang H: LncRNA MALAT1 enhances oncogenic activities of EZH2 in castration-resistant prostate cancer. Oncotarget 2015, 6(38): 41045-41055

26. Li Z, Ma Z, Xu X: Long noncoding RNA MALAT1 correlates with cell viability and mobility by targeting miR223p in renal cell carcinoma via the PI3K/Akt pathway. Oncol Rep 2019, 41(2): 11131121.

27. Su Y, Lu J, Chen X, Liang C, Luo P, Qin C, Zhang J: Long noncoding RNA HOTTIP affects renal cell carcinoma progression by regulating autophagy via the PI3K/Akt/Atg13 signaling pathway. 2019, 145(3): 573-588.

28. Yao J, Chen Y, Wang Y, Liu S, Yuan X, Pan F, Geng P: Decreased expression of a novel lncRNA CADM1-AS1 is associated with poor prognosis in patients with clear cell renal cell carcinomas. Int J Clin Exp Pathol 2014, 7(6): 2758-2767. 
29. Xue S, Li QW, Che JP, Guo Y, Yang FQ, Zheng JH: Decreased expression of long non-coding RNA NBAT-1 is associated with poor prognosis in patients with clear cell renal cell carcinoma. Int J Clin Exp Pathol 2015, 8(4): 3765-3774.

30. Yan C, Jiang Y, Wan Y, Zhang L, Liu J, Zhou S, Cheng W: Long noncoding RNA NBAT-1 suppresses tumorigenesis and predicts favorable prognosis in ovarian cancer. Onco Targets Ther 2017, 10: 1993-2002.

31. Ellinger J, Alam J, Rothenburg J, Deng M, Schmidt D, Syring I, Miersch H, Perner S, Muller SC: The long non-coding RNA lncZNF180-2 is a prognostic biomarker in patients with clear cell renal cell carcinoma. Am J Cancer Res 2015, 5(9): 2799-2807.

32. Liu H, Chen P, Jiang C, Han J, Zhao B, Ma Y, Mardan M: Screening for the Key lncRNA Targets Associated With Metastasis of Renal Clear Cell Carcinoma. Medicine (Baltimore) 2016, 95(2): e2507.

33. Sakurai K, Reon BJ, Anaya J, Dutta A: The lncRNA DRAIC/ PCAT29 Locus Constitutes a Tumor-Suppressive Nexus. Mol Cancer Res 2015, 13(5): 828-838.

34. Xu S, Wang P, You Z, Meng H, Mu G, Bai X, Zhang G, Zhang J, Pang D: The long non-coding RNA EPB41L4A-AS2 inhibits tumor proliferation and is associated with favorable prognoses in breast cancer and other solid tumors. Oncotarget 2016, 7(15): 20704-20717.

35. Huang T, Wang X, Yang X, Ji J, Wang Q, Yue X, Dong Z: Long Non-Coding RNA DUXAP8 Enhances Renal Cell Carcinoma Progression via Downregulating miR-126. Med Sci Monit 2018, 24 : 7340-7347.

36. Verma SS, Rahman MH, Deyholos MK, Basu U, Kav NNV: Differential Expression of miRNAs in Brassica napus Root following Infection with Plasmodiophora brassicae. PLOS ONE 2014, 9(1): e86648.

37. Parpart S, Wang XW: microRNA Regulation and Its Consequences in Cancer. Current pathobiology reports 2013, 1(1): 71-79.

38. Zhang J, Ye Y, Chang DW, Lin SH, Huang M, Tannir NM, Matin S, Karam JA, Wood CG, Chen ZN et al: Global and Targeted miRNA Expression Profiling in Clear Cell Renal Cell Carcinoma Tissues Potentially Links miR-155-5p and miR-210-3p to both Tumorigenesis and Recurrence. Am J Pathol 2018, 188(11): 2487-2496.

39. Wang X, Chen X, Han W, Ruan A, Chen L, Wang R, Xu Z, Xiao P, Lu X, Zhao Y et al: miR-200c Targets CDK2 and Suppresses Tumorigenesis in Renal Cell Carcinoma. Mol Cancer Res 2015, 13(12): 1567-1577.

40. Kanwal R, Plaga AR, Liu X, Shukla GC, Gupta S: MicroRNAs in prostate cancer: Functional role as biomarkers. Cancer Letters 2017, 407: 9-20

41. McCormick RI, Blick C, Ragoussis J, Schoedel J, Mole DR, Young AC, Selby PJ, Banks RE, Harris AL: miR-210 is a target of hypoxiainducible factors 1 and 2 in renal cancer, regulates ISCU and correlates with good prognosis. British Journal Of Cancer 2013, 108: 1133 .

42. Kristensen LS, Hansen TB, Veno MT, Kjems J: Circular RNAs in cancer: opportunities and challenges in the field. Oncogene 2018, 37(5): 555-565.

43. Hansen TB, Jensen TI, Clausen BH, Bramsen JB, Finsen B, Damgaard CK, Kjems J: Natural RNA circles function as efficient microRNA sponges. Nature 2013, 495(7441): 384-388

44. Zhong Y, Du Y, Yang X, Mo Y, Fan C, Xiong F, Ren D, Ye X, Li C, Wang Y et al: Circular RNAs function as ceRNAs to regulate and control human cancer progression. Molecular cancer 2018, 17(1): 7979.

45. Dudekula DB, Panda AC, Grammatikakis I, De S, Abdelmohsen K, Gorospe M: CircInteractome: A web tool for exploring circular RNAs and their interacting proteins and microRNAs. RNA biology 2015, 13(1): 34-42.

46. Liu J, Liu T, Wang X, He A: Circles reshaping the RNA world: from waste to treasure. Molecular Cancer 2017, 16(1): 58.

47. Li J, Yang J, Zhou P, Le Y, Zhou C, Wang S, Xu D, Lin HK, Gong
Z: Circular RNAs in cancer: novel insights into origins, properties, functions and implications. Am J Cancer Res 2015, 5(2): 472-480.

48. Zheng Q, Bao C, Guo W, Li S, Chen J, Chen B, Luo Y, Lyu D, Li Y, Shi G et al: Circular RNA profiling reveals an abundant circHIPK3 that regulates cell growth by sponging multiple miRNAs. Nat Commun 2016, 7: 11215.

49. Chen B, Wang C, Zhang J, Zhou Y, Hu W, Guo T: New insights into long noncoding RNAs and pseudogenes in prognosis of renal cell carcinoma. Cancer Cell Int 2018, 18: 157.

50. Zhou B, Zheng P, Li Z: CircPCNXL2 sponges miR-153 to promote the proliferation and invasion of renal cancer cells through upregulating ZEB2. Cell Cycle 2018, 17(23): 2644-2654.

51. Han Z, Zhang Y, Sun Y, Chen J, Chang C, Wang X, Yeh S: ERbetaMediated Alteration of circATP2B1 and miR-204-3p Signaling Promotes Invasion of Clear Cell Renal Cell Carcinoma. Cancer Res 2018, 78(10): 2550-2563.

52. Li M, Wang Y, Song Y, Bu R, Yin B, Fei X, Guo Q, Wu B: MicroRNAs in renal cell carcinoma: a systematic review of clinical implications (Review). Oncol Rep 2015, 33(4): 1571-1578.

53. Hou J, Jiang W, Zhu L, Zhong S, Zhang H, Li J, Zhou S, Yang S, He Y, Wang D et al: Circular RNAs and exosomes in cancer: a mysterious connection. 2018, 20(9): 1109-1116.

54. Chen D, Chen W, Xu Y, Zhu M, Xiao Y, Shen Y, Zhu S, Cao C, Xu $\mathrm{X}$ : Upregulated immune checkpoint HHLA2 in clear cell renal cell carcinoma: a novel prognostic biomarker and potential therapeutic target. J Med Genet 2019, 56(1): 43-49.

55. Xiong Y, Zhang J, Song C: CircRNA ZNF609 functions as a competitive endogenous RNA to regulate FOXP4 expression by sponging miR-138-5p in renal carcinoma. 2018, https://doi. org/10.1002/jcp.27744. Epub ahead of print.

56. Lerner I, Bartok O, Wolfson V, Menet JS, Weissbein U, Afik S, Haimovich D, Gafni C, Friedman N, Rosbash M et al: Clk posttranscriptional control denoises circadian transcription both temporally and spatially. Nat Commun 2015, 6: 7056

57. Chen S, Ma P, Li B, Zhu D, Chen X, Xiang Y, Wang T, Ren X, Liu C, Jin X: LncRNA CCAT1 inhibits cell apoptosis of renal cell carcinoma through up-regulation of Livin protein. Mol Cell Biochem 2017, 434(1-2): 135-142.

58. He HT, Xu M, Kuang Y, Han XY, Wang MQ, Yang Q: Biomarker and competing endogenous RNA potential of tumor-specific long noncoding RNA in chromophobe renal cell carcinoma. Onco Targets Ther 2016, 9: 6399-6406.

59. Song J, Peng J, Zhu C, Bai G, Liu Y, Zhu J, Liu J: Identification and Validation of Two Novel Prognostic lncRNAs in Kidney Renal Clear Cell Carcinoma. Cell Physiol Biochem 2018, 48(6): 2549-2562.

60. Jin L, Quan J, Pan X, He T, Hu J, Li Y, Gui Y, Yang S, Mao X, Chen $\mathrm{Y}$ et al: Identification of lncRNA EGOT as a tumor suppressor in renal cell carcinoma. Mol Med Rep 2017, 16(5): 7072-7079.

61. He W, Zhong G, Wang P, Jiang C, Jiang N, Huang J: Downregulation of long noncoding RNA FENDRR predicts poor prognosis in renal cell carcinoma. Oncol Lett 2019, 17(1): 103-112.

62. Xiao ZD, Han L: Energy stress-induced lncRNA FILNC1 represses c-Myc-mediated energy metabolism and inhibits renal tumor development. Nature Communication 2017, 8(1): 783.

63. Wang L, Cai Y, Zhao X, Jia X, Zhang J, Liu J, Zhen H, Wang T, Tang $\mathrm{X}$, Liu Y et al: Down-regulated long non-coding RNA H19 inhibits carcinogenesis of renal cell carcinoma. Neoplasma 2015, 62(3): 412418.

64. Wu Y, Tan C, Weng WW, Deng Y, Zhang QY, Yang XQ, Gan HL, Wang T, Zhang PP, Xu MD et al: Long non-coding RNA Linc00152 is a positive prognostic factor for and demonstrates malignant biological behavior in clear cell renal cell carcinoma. Am J Cancer Res 2016, 6(2): 285-299.

65. Zheng Z, Zhao F, Zhu D, Han J, Chen H, Cai Y, Chen Z, Xie W: Long Non-Coding RNA LUCAT1 Promotes Proliferation and Invasion in Clear Cell Renal Cell Carcinoma Through AKT/GSK- 
3beta Signaling Pathway. Cell Physiol Biochem 2018, 48(3): 891-904.

66. Ning L, Li Z, Wei D, Chen H, Yang C: LncRNA, NEAT1 is a prognosis biomarker and regulates cancer progression via epithelialmesenchymal transition in clear cell renal cell carcinoma. Cancer Biomark 2017, 19(1): 75-83.

67. Wang G, Zhang ZJ, Jian WG, Liu PH, Xue W, Wang TD, Meng YY, Yuan C, Li HM, Yu YP et al: Novel long noncoding RNA OTUD6BAS1 indicates poor prognosis and inhibits clear cell renal cell carcinoma proliferation via the Wnt/beta-catenin signaling pathway. Mol Cancer 2019, 18(1): 15.

68. Yang T, Zhou H, Liu P, Yan L, Yao W, Chen K, Zeng J, Li H, $\mathrm{Hu}$ J, Xu H et al: IncRNA PVT1 and its splicing variant function as competing endogenous RNA to regulate clear cell renal cell carcinoma progression. Oncotarget 2017, 8(49): 85353-85367.

69. Shi J, Zhang W, Tian H, Zhang Q, Men T: IncRNA ROR promotes the proliferation of renal cancer and is negatively associated with favorable prognosis. Mol Med Rep 2017, 16(6): 9561-9566.

70. Zhai W, Sun Y, Guo C, Hu G, Wang M, Zheng J, Lin W, Huang Q, Li $\mathrm{G}$, Zheng J et al: LncRNA-SARCC suppresses renal cell carcinoma (RCC) progression via altering the androgen receptor(AR)/miRNA143-3p signals. Cell Death Differ 2017, 24(9): 1502-1517.

71. Gong X, Siprashvili Z, Eminaga O, Shen Z, Sato Y, Kume H, Homma Y, Ogawa S, Khavari PA, Pollack JR et al: Novel lincRNA SLINKY is a prognostic biomarker in kidney cancer. Oncotarget 2017, 8(12): 18657-18669.

72. Wang PQ, Wu YX, Zhong XD, Liu B, Qiao G: Prognostic significance of overexpressed long non-coding RNA TUG1 in patients with clear cell renal cell carcinoma. Eur Rev Med Pharmacol Sci 2017, 21(1): 82-86.

73. Li Y, Wang T, Li Y, Chen D, Yu Z, Jin L, Ni L, Yang S, Mao X, Gui $Y$ et al: Identification of long-non coding RNA UCA1 as an oncogene in renal cell carcinoma. Mol Med Rep 2016, 13(4): 33263334 .

74. Sun K, Jia Z, Duan R, Yan Z, Jin Z, Yan L, Li Q, Yang J: Long noncoding RNA XIST regulates miR-106b-5p/P21 axis to suppress tumor progression in renal cell carcinoma. Biochem Biophys Res Commun 2019, 510(3): 416-420.

75. Ma PJ, Guan QK, Xu DW, Zhao J, Qin N, Jin BZ: LncRNA PANDAR as a prognostic marker in Chinese cancer. Clin Chim Acta 2017, 475: 172-177.

76. Xu Z, Yang F, Wei D, Liu B, Chen C, Bao Y, Wu Z, Wu D, Tan H, $\mathrm{Li} \mathrm{J}$ et al: Long noncoding RNA-SRLR elicits intrinsic sorafenib resistance via evoking IL-6/STAT3 axis in renal cell carcinoma. Oncogene 2017, 36(14): 1965-1977.

77. He X, Liu H, Guo F, Feng Y, Gao Y, Sun F, Song B, Lu H, Li Y: Long non-coding RNA Z38 promotes cell proliferation and metastasis in human renal cell carcinoma. Mol Med Rep 2017, 16(4): 5489-5494.

78. Cao Y, Xu R, Xu X, Zhou Y, Cui L, He X: Downregulation of lncRNA CASC2 by microRNA-21 increases the proliferation and migration of renal cell carcinoma cells. Mol Med Rep 2016, 14(1): 1019-1025.

79. Ni W, Song E, Gong M, Li Y, Yao J, An R: Downregulation of lncRNA SDPR-AS is associated with poor prognosis in renal cell carcinoma. Onco Targets Ther 2017, 10: 3039-3047.

80. Peng F, Shi X, Meng Y, Dong B, Xu G, Hou T, Shi Y, Liu T: Long non-coding RNA HOTTIP is upregulated in renal cell carcinoma and regulates cell growth and apoptosis by epigenetically silencing of LATS2. Biomed Pharmacother 2018, 105: 1133-1140. 